\title{
Low b-value diffusion weighted imaging is promising in the diagnosis of brain death and hypoxic-ischemic injury secondary to cardiopulmonary arrest
}

\author{
Miriam E. Peckham ${ }^{1,4^{*}} \mathbb{D}$, Jeffrey S. Anderson', Ulrich A. Rassner', Lubdha M. Shah', Peter J. Hinckley', \\ Adam de Havenon'2, Seong-Eun $\mathrm{Kim}^{3}$ and J. Scott McNally ${ }^{1}$
}

\begin{abstract}
Background: Cardiorespiratory arrest can result in a spectrum of hypoxic ischemic brain injury leading to global hypoperfusion and brain death (BD). Because up to $40 \%$ of patients with BD are viable organ donors, avoiding delayed diagnosis of this condition is critical. High b-value diffusion weighted imaging (DWI) measures primarily molecular self-diffusion; however, low b-values are sensitive to perfusion. We investigated the feasibility of low bvalue DWI in discriminating the global hypoperfusion of BD and hypoxic ischemic encephalopathy (HIE).

Methods: We retrospectively reviewed cardiorespiratory arrest subjects with a diagnosis of HIE or BD. Inclusion criteria included brain DWI acquired at both low $\left(50 \mathrm{~s} / \mathrm{mm}^{2}\right)$ and high $\left(1000-2000 \mathrm{~s} / \mathrm{mm}^{2}\right)$ b-values. Automated segmentation was used to determine mean b50 apparent diffusion coefficient (ADC) values in gray and white matter regions. Normal subjects with DWI at both values were used as age- and sex-matched controls.

Results: We evaluated 64 patients (45 with cardiorespiratory arrest and 19 normal). Cardiorespiratory arrest patients with BD had markedly lower mean b50 ADC in gray matter regions compared with HIE $\left(0.70 \pm 0.18 \mathrm{vs} .1 .95 \pm 0.25 \times 10^{-3} \mathrm{~mm}^{2} / \mathrm{s}\right.$, $p<0.001$ ) and normal subjects (vs. $1.79 \pm 0.12 \times 10^{-3} \mathrm{~mm}^{2} / \mathrm{s}, p<0.001$ ). HIE had higher mean b50 ADC compared with normal $\left(1.95 \pm 0.25\right.$ vs. $\left.1.79 \pm 0.12 \times 10^{-3} \mathrm{~mm}^{2} / \mathrm{s}, p=0.016\right)$. There was wide separation of gray matter ADC values in BD subjects compared with age matched normal and HIE subjects. White matter values were also markedly decreased in the BD population, although they were less predictive than gray matter.
\end{abstract}

Conclusion: Low b-value DWI is promising for the discrimination of HIE with maintained perfusion and brain death in cardiorespiratory arrest.

Keywords: Brain death, Hypoxic ischemic encephalopathy, Cardiorespiratory arrest, Diffusion imaging

\section{Background}

Evaluation of brain perfusion is critically important in patients after cardiorespiratory arrest. These patients suffer an initial hypoxic ischemic injury which can lead to cytotoxic edema as well as global hypoperfusion and brain death (BD). In these patients, ancillary tests can evaluate perfusion status when the clinical examination

\footnotetext{
* Correspondence: Miriam.Peckham@hsc.utah.edu

'Department of Radiology and Imaging Sciences, University of Utah, Salt Lake City, UT, USA

${ }^{4}$ Department of Radiology and Imaging Sciences, University of Utah Health Sciences Center, 30 North, 1900 East \#1A071, Salt Lake City, UT 84132-2140, USA Full list of author information is available at the end of the article
}

is limited [1]. Over $40 \%$ of subjects with $\mathrm{BD}$ are viable organ donors; however, only $10 \%$ of subjects with cardiac arrest go on to BD. Because delayed diagnosis of $\mathrm{BD}$ has been found to contribute to the overall shortage of viable organ transplants, some have argued for earlier use of ancillary testing in subjects where examination in this critical diagnosis is obscured by central nervous system (CNS) depressant medication $[2,3]$.

Introduction of targeted temperature management has significantly improved the clinical course in comatose cardiorespiratory arrest patients, with some patients being discharged with minimal brain damage. However,

(c) The Author(s). 2018 Open Access This article is distributed under the terms of the Creative Commons Attribution 4.0 International License (http://creativecommons.org/licenses/by/4.0/), which permits unrestricted use, distribution, and 
neurologic assessment during this time has remained a critical barrier since sedation and muscle paralysis from hypothermia obscure the clinical examination in the first 24-48 h, a crucial time for these patients $[4,5]$. It is suspected that sedation-obscured clinical examination may contribute to withdrawal of life-sustaining therapy in up to $20 \%$ of patients during this time who may have otherwise had complete neurologic recovery $[4,5]$. Conversely, CNS depressant medications have also been linked to a delay in prognosis of patients with $\mathrm{BD}$, which has negative implications for organ transplantation viability and has led to increased mortality in cardiac transplant recipients [2]. These two extremes demonstrate the clinical dilemma in these unresponsive patients under hypothermic therapy or CNS depressants: the discrimination between subjects sustaining global injury with maintained cerebral blood flow, and subjects with cessation of blood flow and irreversible loss of function defined as BD [6].

Single photon emission computed tomography (SPECT) nuclear scintigraphy is an accepted ancillary test for BD [7-12]. Transcranial Doppler has also been used as a prognostic tool, showing poor outcomes for patients with hypoperfusion, and good prediction of survivability in patients with normal perfusion [13, 14]. Computed tomography angiography (CTA) has been found to be comparable to ancillary tests, but lacks sensitivity [1]. In magnetic resonance imaging (MRI), the presence of bilateral transcerebral and cortical vein signs on susceptibility-weighted imaging (SWI) are helpful indicators in patients with both BD and hypoxic ischemic encephalopathy (HIE), although they are not specific [7, 15]. MRI in BD also consistently shows loss of vascular flow-voids on T2 spin echo and flow-related enhancement on magnetic resonance angiography (MRA), and diffuse cerebral edema with sulcal effacement [7]. Arterial spin labeling (ASL) has been validated as an effective noncontrast method for demonstrating perfusion deficits by demonstrating global hypoperfusion in $\mathrm{BD}$ with diffusely impaired cerebral blood flow on visual and quantitative analysis [16, 17]. Diffusion weighted imaging (DWI) shows promise, and apparent diffusion coefficient (ADC) values obtained at high gradient strengths (b750-2000) have been shown to be helpful prognostic indicators in patients with HIE, with decreased ADC values predicting poorer outcomes [18-20]. Still, current use of high b-value DWI does not quantify perfusion and cannot be used to discriminate between the cellular injury of HIE and hypoperfusion related to BD.

DWI measures hydrogen motion in tissues. In addition to intra-/extracellular water self-diffusion, DWI is also affected by bulk water movement (cerebrospinal fluid (CSF) and intravascular motion [21-23]. While high b-value DWI measures molecular self-diffusion, low b-values are more sensitive to blood flow [21, 23-25].
This is the basis of intravoxel incoherent motion (IVIM), a noncontrast sequence allowing derivation of perfusion (vascular) and diffusion (extravascular) fractions and is quantified by ADC [23] which has been used to evaluate acute stroke, dementia, as well as brain and head and neck masses [26-32]. Though IVIM has many advantages, including its independence from arterial timing and its ability to quantify perfusion without contrast, postprocessing is time consuming and imaging times can be long since multiple b-values are necessary for processing $[26,33]$. The current view is that b-value gradients between 0 and $200 \mathrm{~s} / \mathrm{mm}^{2}$ primarily measure perfusion, whereas gradient strengths greater than $200 \mathrm{~s} /$ $\mathrm{mm}^{2}$ primarily measure molecular diffusion $[22,34]$.

We hypothesized that low b-value DWI could also be a helpful diagnostic tool in detecting global hypoperfusion. Specifically, our goal was to determine if low b-value DWI could separate the cellular injury of HIE from the global hypoperfusion of BD. To test this hypothesis, we evaluated patients after cardiorespiratory arrest undergoing MRI with low b-value DWI.

\section{Methods}

This retrospective study was conducted under an Institutional Review Board approved protocol and informed consent was waived. Investigators were compliant with the Health Insurance Portability and Accountability Act and Good Clinical Practice guidelines.

\section{Subjects}

An MRI report search was initiated on all cardiorespiratory arrest patients diagnosed with HIE or BD at our quaternary care center from 2012 to 2018. Inclusion criteria included findings of diffuse hypoxic and/or anoxic injury on MRI and clinical history of arrest. A b50 DWI sequence has been routinely added at our institution as a rapid noncontrast method of perfusion evaluation in patients with stroke within the last 6 years. Only subjects with DWI acquired at both low b-values of $50 \mathrm{~s} / \mathrm{mm}^{2}$ in three orthogonal planes $(\mathrm{TR}=8000 \mathrm{~ms}, \mathrm{TE}=90 \mathrm{~ms}$, flip angle $=90$ degrees, resolution $=2.2 \times 2.2 \times 11 \mathrm{~mm}$, slice gap $=1 \mathrm{~mm}$, time $=1 \mathrm{~min} 47 \mathrm{~s}$ ), and high b-values of $1000-2000 \mathrm{~s} / \mathrm{mm}^{2}$ in 12 to 20 diffusion direction planes (resolution $=1.8 \times 1.8 \times 3.3 \mathrm{~mm}$ ), as well as high-resolution magnetization prepared rapid acquisition gradient echo (MPRAGE) $(\mathrm{TR}=1530 \mathrm{~ms}, \mathrm{TE}=4.04 \mathrm{~ms}$, $\mathrm{TI}=950 \mathrm{~ms}$, flip angle $=15$ degrees, resolution $=1.0 \times$ $1.0 \times 1.2 \mathrm{~mm}$ ) were included in this study. Subjects were excluded for the following: no MRI evidence of hypoxia, primarily embolic disease, the presence of a large vessel infarct, extensive motion or metallic artifact causing image degradation, and lack of or unusable b50 $\mathrm{ADC}$ sequence. A formal clinical $\mathrm{BD}$ examination was required in all BD subjects. For comparison, MRIs of 
normal age- and sex-matched subjects were also collected. All cases were scanned on Siemens 1.5 T scanners (Avanto or Aera).

\section{Clinical review}

Each subject underwent a chart review with the following information recorded: age, sex, cause of arrest, time between arrest and return of spontaneous circulation (ROSC), and time between ROSC and MRI. The use of targeted temperature management was recorded for each subject. The blood pressure and EEG findings closest to the time of MRI were recorded. In the case of patient survival, the cerebral performance category was determined.

\section{Voxel segmentation}

To obtain estimates of b50 ADC values localized to brain gray and white matter, MPRAGE segmentation was performed using SPM 12 (Wellcome Trust, London) for MATLAB (Natick, MA). The MPRAGE image was coregistered using rigid transform to the b50 ADC scan for each subject (coregister: estimate) and the MPRAGE image was segmented into gray matter, white matter, and CSF components (segment: 3 tissue classes, thorough clean, native space). The white matter and gray matter images were resampled at the resolution of the coregistered b50 ADC image, and voxels for gray and white matter were included in a restriction mask. ADC values for these voxels were obtained for each BD, HIE, and control subject, and histograms and summary statistics were obtained for these voxels, with scaling of the histogram to peak value (bin size 0.01 from 0 to 3 for b50 ADC value). The ADC values were calculated for gray and white matter at the time of imaging using Siemens online reconstruction algorithm and reported in units of $1000 \times \mathrm{ADC}$, so $\mathrm{ADC}$ values obtained from the images were divided by 1000 prior to analysis.

\section{Statistical analysis}

Two-tailed $t$ tests were used to determine significance at $p<0.05$, and logistic regression analyses were performed using Stata 14 (Stata Statistical Software: Release 14; College Station, TX).

\section{Results \\ Subjects}

A total of 98 subjects with a clinical history of cardiorespiratory arrest received a brain MRI at our institution from 2012 to 2018. Of these subjects, 53 were excluded from the analysis for the following reasons: 16 demonstrated predominantly embolic disease, 7 had the presence of a large-vessel infarct, 4 had image degradation related to artifact, 8 lacked a b50 ADC sequence, 16 had a normal MRI with no evidence of hypoxic disease, and 2 had MRI and clinical findings consistent with BD but they never underwent a formal BD examination. Forty-five cardiorespiratory arrest subjects met all inclusion criteria for analysis, with all containing b50 ADC imaging and high b-value DWI imaging (36 subjects with b2000, and 9 subjects with b1000 DWI). Of these, 9/45 had a clinical diagnosis of BD and 36/45 HIE (Table 1).

The nine BD subjects included in the study all underwent formal clinical BD examination and, in addition, a board-certified neurologist with experience in BD testing reviewed the patient charts, blinded to neuroimaging findings, to determine the diagnosis of BD using the 2010 American Academy of Neurology criteria [35]. The following findings were documented: examination performed without the presence of CNS depressant medications,

Table 1 Demographic and clinical information of the three subject groups

\begin{tabular}{|c|c|c|c|}
\hline Subject demographics & Brain death & HIE & Normal \\
\hline$n$ & 9 & 36 & 19 \\
\hline Age, years & $27-68(44 \pm 15.9)$ & $22-64(44 \pm 16.5)$ & $21-64(45 \pm 15.1)$ \\
\hline Sex, female $(F)$, male $(M)$ & $3 \mathrm{~F}, 6 \mathrm{M}$ & $15 \mathrm{~F}, 21 \mathrm{M}$ & $10 \mathrm{~F}, 9 \mathrm{M}$ \\
\hline Cardiorespiratory arrest $(n)$ & $9 / 9$ & $36 / 36$ & $0 / 19$ \\
\hline Time to ROSC & $3.5-40 \min (29.2 \pm 13.2)$ & $3-60 \min 16.4 \pm 15.0)$ & N/A \\
\hline Time from ROSC to MRI & $0.1-6$ days $(2.2 \pm 1.8)$ & $0.2-16$ days $(3.8 \pm 3.0)$ & N/A \\
\hline \multicolumn{4}{|l|}{ Blood pressure closest to scan time } \\
\hline Systolic & $81-184(118.9 \pm 33.1)$ & $91-164(127.5 \pm 21.1)$ & $110-144(127.8 \pm 11.5)$ \\
\hline Diastolic & $52-141(77.5 \pm 28.1)$ & $53-109(73.6 \pm 15.8)$ & 43-91 (75.5 \pm 14.1$)$ \\
\hline Survival (n) & $0 / 9$ & $7 / 36$ & N/A \\
\hline b50 ADC - gray matter (in $\mathrm{ADC} \times 10^{-3} \mathrm{~mm}^{2} / \mathrm{s}$ ) & $0.70 \pm 0.18$ & $1.95 \pm 0.25$ & $1.79 \pm 0.12$ \\
\hline b50 ADC - white matter (in ADC $\times 10^{-3} \mathrm{~mm}^{2} / \mathrm{s}$ ) & $0.50 \pm 0.17$ & $1.44 \pm 0.24$ & $1.26 \pm 0.14$ \\
\hline
\end{tabular}

Values are shown as range (mean \pm standard deviation) or mean \pm standard deviation unless otherwise indicated

$A D C$ apparent diffusion coefficient, HIE hypoxic ischemic encephalopathy, MRI magnetic resonance imaging, N/A not applicable, ROSC return of spontaneous circulation 
pupils fixed and dilated, no corneal reflex, no withdrawal to pain or gag reflex, and no return of neurological function. In two subjects the apnea test was undocumented, and in one subject absence of brainstem reflexes was undocumented on chart review. In two subjects ancillary testing was required and SPECT nuclear scintigraphy was performed to confirm the BD diagnosis.

All HIE subjects had clinical and imaging diagnosis of HIE. Nineteen normal subjects underwent MRI for subjective neurologic symptoms ranging from headache to vertigo. All had a negative neurological and imaging work-up with normal MRI findings for age.

$T$ tests demonstrated no significant age difference between BD versus normal $(p=0.98)$ or $\operatorname{HIE}(p=0.122)$ groups. Fisher's exact test also showed no difference in sex proportions between $\mathrm{BD}$ versus HIE $(p=0.72)$ or normal $(p=0.43)$ subjects.

There was a poor correlation of time to ROSC and ADC values $(r=-0.27$ in gray matter and -0.29 in white matter) and time between ROSC and MRI with ADC values ( $r=0.23$ in gray matter and 0.27 in white matter). ADC values between gray and white matter regions demonstrated excellent correlation $(r=0.98)$.

\section{Clinical results}

The majority of HIE and BD subjects had a cardiac etiology for arrest (30/45 patients). The remaining subjects had mixed cardiopulmonary or predominantly pulmonary causes for arrest including asthma exacerbation, hanging, status epilepticus, and overdose.

Time from arrest to ROSC was available in $7 / 9$ subjects with BD and 24/36 subjects with HIE. Average time to ROSC was longer in the BD population $(29.2 \mathrm{~min}) \mathrm{com}$ pared with the HIE population (16.4 $\mathrm{min}, p=0.13$ ) (Table 1). Time between ROSC and MRI was shorter in the BD population (2.2 days) compared with HIE (3.8 days, $p=0.3)$. Information on the targeted therapeutic protocol was available in 35/36 HIE patients, with 28 of these patients undergoing this therapy. In the BD population, 7/9 subjects underwent targeted temperature management.

EEG was performed in 32/36 subjects with HIE and demonstrated predominantly global slowing in 23 subjects, with the remainder of subjects having a burst suppression pattern, as well as a myoclonic seizure pattern. Seven BD subjects underwent EEG with all seven demonstrating a flat pattern.

Seven HIE subjects survived after the acute hospital course, with two of these subjects having a Cerebral Performance Category (CPC) score of 2 (good function), and five patients demonstrating a CPC score of 3 or 4 (poor function). There was no significant difference in ADC values between the surviving subjects and the remaining population who died $(p=0.19)$.

\section{Qualitative MRI findings}

All BD subjects had diffuse gray matter hypointensity on the b50 ADC maps (Fig. 1a), and 8/9 subjects had diffuse white matter hypointensity. Additional findings supporting BD included global cerebral edema with sulcal effacement, tonsillar herniation, and cellular (high b-value) diffusion restriction consistent with anoxic brain injury, with complete loss of flow voids at the skull base on the T2 weighted spin-echo sequence in 7 out of 9 subjects. In the 2/9 subjects with flow voids, one patient had a very thin residual flow voids in the left parasellar internal carotid artery and bilateral M1 segments, and the second patient had more prominent bilateral parasellar internal carotid artery flow voids with flow extending to the bilateral M3 segments.

All HIE subjects had evidence of molecular diffusion restriction on high b-value (1000 or 2000) DWI. Signal abnormality varied between predominantly cortically based restriction in 11 subjects, predominantly deep gray

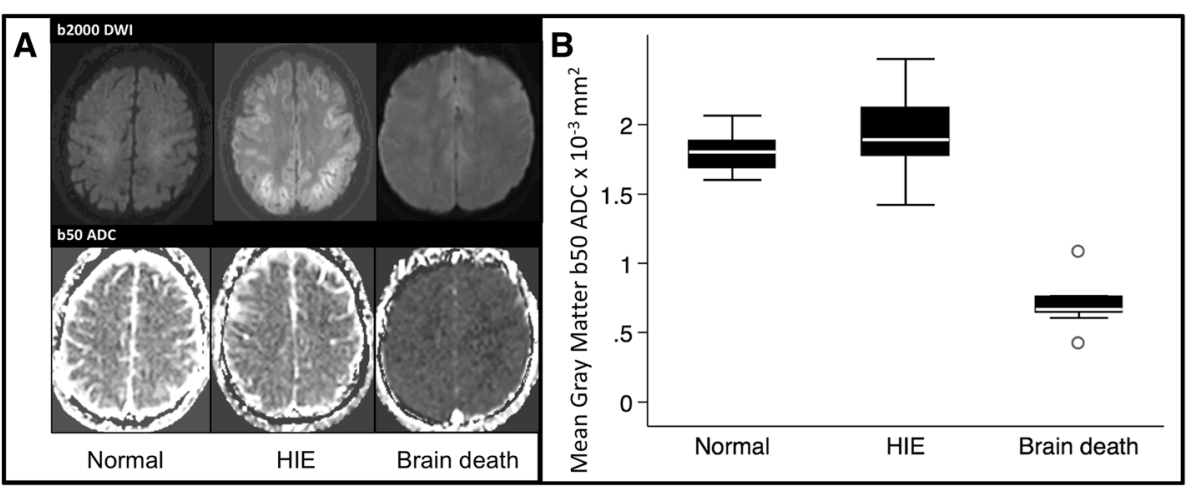

Fig. 1 b50 apparent diffusion coefficient (ADC) values in normal, hypoxic ischemic encephalopathy (HIE), and brain death populations. A panel of representative images (a) demonstrates high b-value DWI trace images (top) and b50 ADC maps (bottom). In brain death patients, there was diffuse hypointensity on ADC maps compared with normal and HIE subjects. Pooled quantified ADC data (b) in box-and-whisker format demonstrate markedly lower mean ADC values in brain death subject gray matter compared with normal and HIE subjects, $p<0.001$ 
structure involvement in 5 subjects, and equal involvement of both regions in 21 subjects. Three HIE subjects had early tonsillar herniation without absence of flow voids. Low b-value DWI was not visually different between HIE and normal subjects (Fig. 1a).

\section{Quantitative differences in ADC values}

Using automated segmentation to quantify ADC values, $\mathrm{BD}$ subjects showed markedly lower mean b50 ADC in gray matter regions compared with $\mathrm{HIE}(0.70 \pm 0.18$ vs. $\left.1.95 \pm 0.25 \times 10^{-3} \mathrm{~mm}^{2} / \mathrm{s}, p<0.001\right)$ and normal subjects (vs. $1.79 \pm 0.12 \times 10^{-3} \mathrm{~mm}^{2} / \mathrm{s}, p<0.001$ ) (Fig. 1b). In white matter regions, BD subjects also demonstrated markedly lower mean b50 ADC compared with HIE $(0.50 \pm 0.17$ vs. $1.44 \pm 0.24, p<0.001)$ and normal subjects $(1.26 \pm 0.14, p<0.001)$. HIE had higher mean b50 ADC compared with normal in gray matter $(1.95 \pm 0.25$ vs. $\left.1.79 \pm 0.12 \times 10^{-3} \mathrm{~mm}^{2} / \mathrm{s}, p=0.016\right)$ and white matter $\left(1.44 \pm 0.24\right.$ vs. $\left.1.26 \pm 0.14 \times 10^{-3} \mathrm{~mm}^{2} / \mathrm{s}, p=0.004\right)$. Histogram analysis demonstrated the b50 ADC values in gray and white matter regions in all subjects, with wide separation of values in the gray matter between BD and normal/HIE subjects (Fig. 2). There was predominantly wide separation in white matter between BD and HIE/ normal subjects with the exception of one BD patient who demonstrated more elevated white matter values (Fig. 2). Logistic regression analysis of mean ADC value prediction of $\mathrm{BD}$ demonstrated perfect prediction with white matter mean $\mathrm{ADC}<0.861 \times 10^{-3} \mathrm{~mm}^{2} / \mathrm{s}$, and perfect prediction with gray matter mean ADC $<1.0858 \times 10^{-3} \mathrm{~mm}^{2} / \mathrm{s}$.

\section{Discussion}

Evaluation of patients after cardiorespiratory arrest is a clinical challenge. We found that using a DWI sequence acquired at a single low b-value on a $1.5 \mathrm{~T}$ magnet was a promising technique for discrimination of BD from HIE and normal perfusion.

Results reinforce the concept that low b-values primarily measure brain perfusion properties, rather than diffusion, as has been previously established in the setting of acute stroke (Fig. 3) [26, 29, 30, 32, 36]. Separation of these properties is best seen in the HIE patients who exhibited profound regions of diffusion restriction on b1000-2000 images indicating cellular injury but showed no perfusion restriction on the b50 sequence, in fact showing elevated ADC values in both gray and white matter regions (Fig. 1).

ASL has successfully confirmed BD both quantitatively and qualitatively; however, the necessity for postprocessing and reliance on proper inflow tagging make it less user friendly and potentially less reliable [16, 24, 37]. Because the b50 sequence is "local" in the sense that it provides perfusion information without reliance on these distant processes, it does not suffer from the same potential inaccuracies.

The b50 DWI sequence also has benefits compared with usage of conventional MRI sequences, as it can give quantitative perfusion information compared with the qualitative evaluation of herniation and flow voids. This was reinforced in our results where the $\mathrm{b} 50 \mathrm{ADC}$ values in gray matter corresponded better with clinical diagnosis of $\mathrm{BD}$ than the presence of flow voids (which were present at the skull base in 2/9 BD subjects). This single low b-value sequence also has benefits in comparison with IVIM, not least of which are its rapid acquisition time, no need for offline postprocessing, and no specific software as it is a simple modification of a standard three-direction diffusion-weighted sequence. While b50 includes both cellular and vascular information, unlike IVIM which separates these components, it is heavily

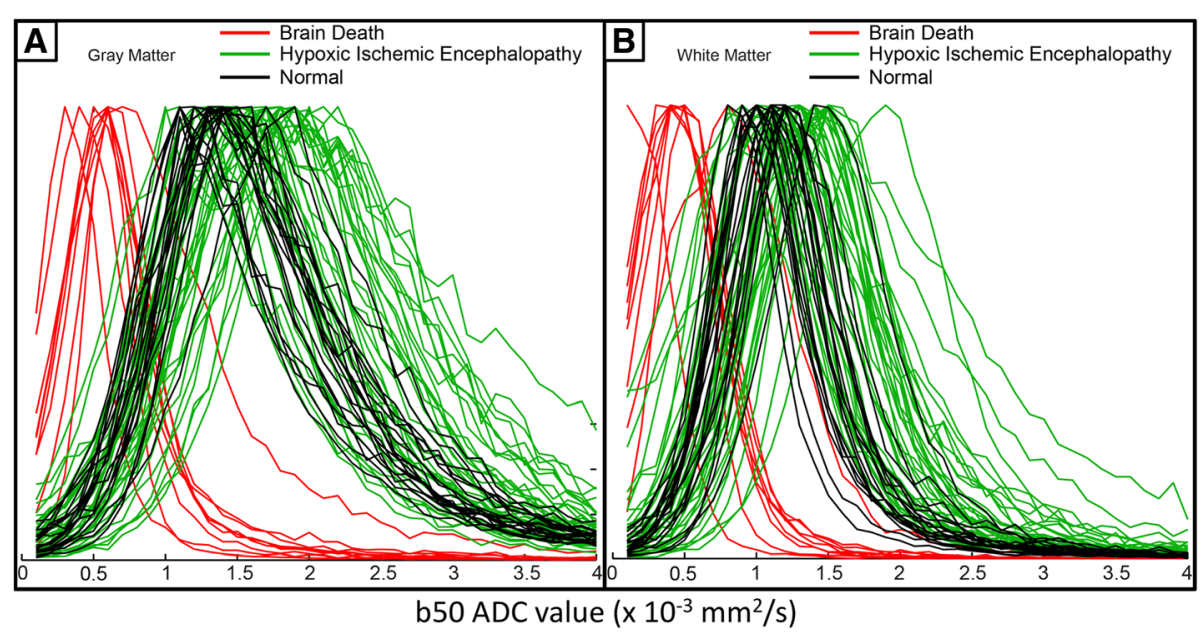

Fig. 2 Histogram analysis of groups. Histogram demonstrating the distribution of apparent diffusion coefficient (ADC) values in brain death subjects (red) from normal (black) and HIE subjects (green) in $\mathbf{a}$ gray and $\mathbf{b}$ white matter regions 


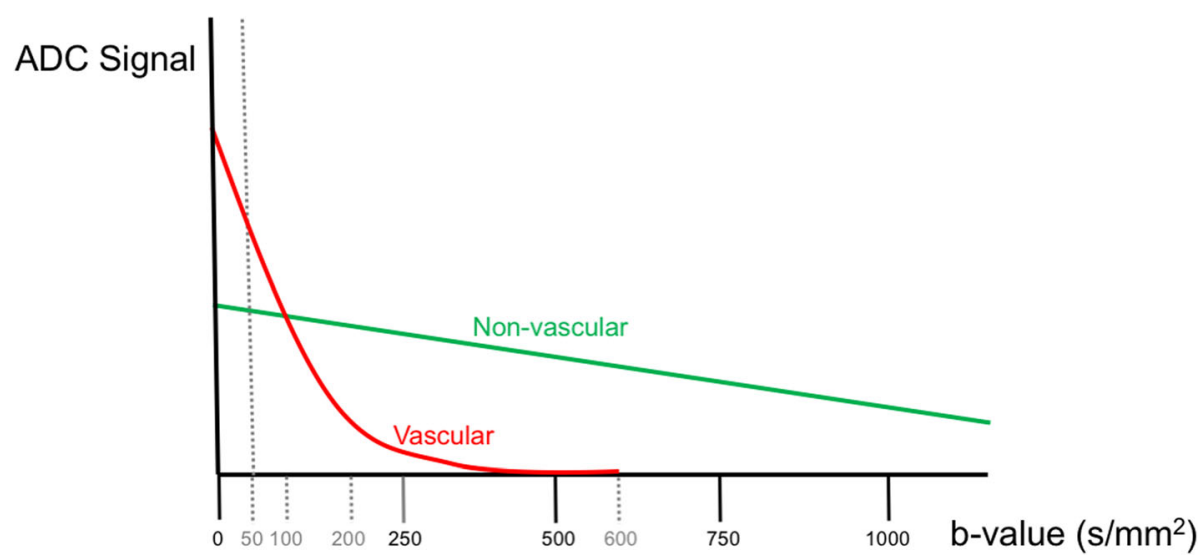

Fig. 3 Perfusion and diffusion contributions to apparent diffusion coefficient (ADC) across a spectrum of b-values. Schematic representation of relative contribution of both vascular and nonvascular motion to total ADC decay. The higher ADC vascular compartment is the primary contributor to total decay at gradient strengths less than $100 \mathrm{~s} / \mathrm{mm}^{2}$, with contribution quickly falling and becoming negligible above a gradient strength of $200 \mathrm{~s} / \mathrm{mm}^{2}$. Perfusion is the primary contributor to ADC at b50

weighted towards perfusion and therefore able to primarily demonstrate perfusion changes. Visually, this sequence had a distinct appearance in BD with diffuse hypointensity from global perfusion restriction (Fig. 1).

Findings suggest that HIE patients have increased perfusion compared with normal controls, in direct contrast to the global hypoperfusion findings in BD. This is in agreement with prior studies demonstrating hyperperfusion with HIE using both ASL and contrast-based perfusion, reflecting the pathophysiologic course of whole-brain insults where initially there is overcompensation of flow to injured areas [38-43].

A single outlier was present in the $\mathrm{BD}$ population which demonstrated more elevated white matter b50 $\mathrm{ADC}$ values (in agreement with $\mathrm{HIE} /$ normal values) than the rest of the $\mathrm{BD}$ subjects (Fig. 2b). This subject also had mildly elevated gray matter values in comparison with the other BD patients; however, these values were still distinctly lower than those in the HIE/normal subjects (Fig. 2a). This subject demonstrated bilateral parasellar carotid flow voids which extended to the bilateral M3 segments on MRI, although all other MRI findings supported BD (diffuse edema, herniation, and diffusion changes consistent with anoxia). Clinically, this subject had a much shorter timeframe from ROSC to MRI than the other BD subjects, being imaged within $2 \mathrm{~h}$ of ROSC as compared with 1-3 days in all other BD patients. The small interval between ROSC and MRI, and the presence of flow voids, support the possibility that this subject had a small residual amount of perfusion at the time of scanning which accounted for the higher b50 ADC values, though no ancillary testing was performed to confirm this. This supports that ADC values in b50 DWI are primarily affected by perfusion, as this patient demonstrated higher values than the $\mathrm{BD}$ patients who demonstrated loss of flow voids at the skull base (Fig. 4). This may give insight into the pattern of perfusion loss in $\mathrm{BD}$, with gray matter regions losing perfusion before white matter, although this is only speculative in such a small study population. These findings support that low b50 ADC values in gray matter are more predictive of clinical BD than those in the white matter, and are more predictive of $\mathrm{BD}$ than the absence of flow voids at the skull base. A larger study population with confirmatory ancillary testing is necessary to further delineate the optimal b50 ADC threshold for this diagnosis.

Study limitations include vulnerability of echo-planar DWI to motion, chemical shift, susceptibility, and N/2 ghost artifacts, and the use of two different $1.5 \mathrm{~T}$ magnets in the study population, all of which could cause inaccuracies in ADC measurements. Additionally, this study is limited by small power and retrospective case-control comparisons. Prospective studies involving larger numbers of BD patients across site, scanner, and sequence architecture are warranted to further establish this technique.

Because BD is primarily a clinical diagnosis, and imaging is only used when the clinical examination cannot be fully obtained or results are in question, the majority of our BD subjects did not have correlative perfusion imaging (7/9). While this is a weakness in terms of validating this particular sequence, low b-value imaging itself has been previously validated as a reliable noncontrast perfusion technique [23-26]. Our study demonstrating wide discrimination of mean ADC values with lack of overlap in the $\mathrm{BD}$ group in comparison with the perfused normal and HIE groups in gray matter serves to further support this technique. Although the clinical examination remains the gold standard for diagnosis of $\mathrm{BD}$, this low b-value technique may be a promising supportive tool and deserves further study. 


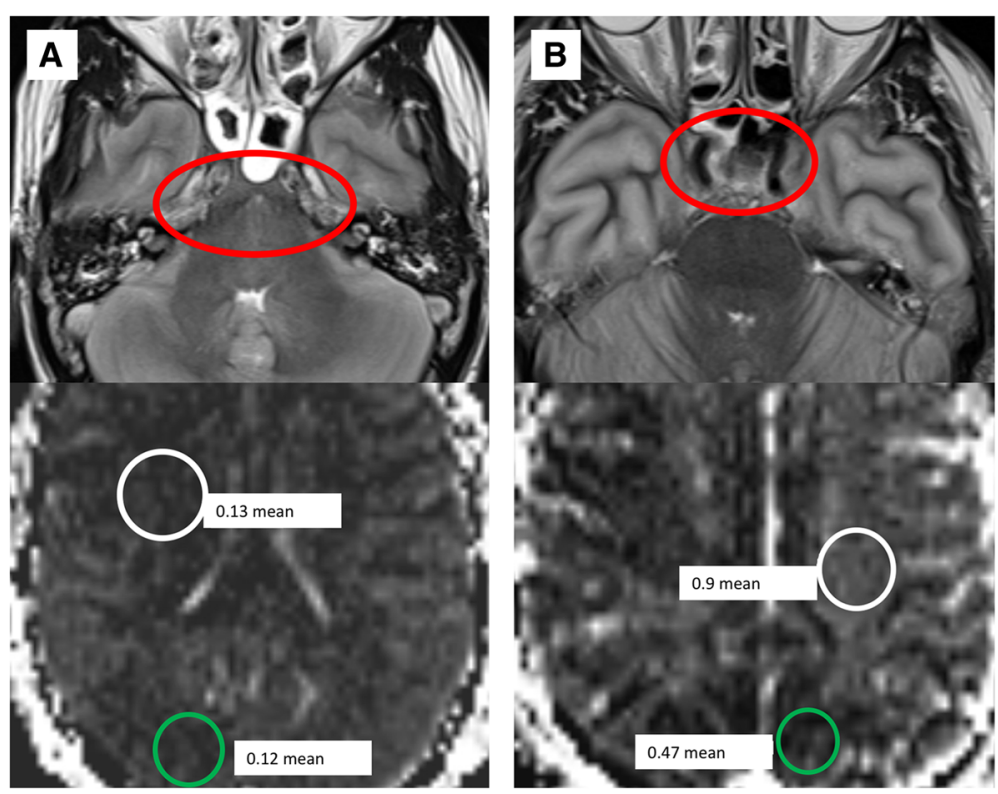

Fig. 4 Correlation of hypointensity on b50 ADC maps in the BD population in comparison with flow voids. T2w (upper row) and b50 ADC (lower row) MRI images in two subjects with $B D$, with a subject $A$ demonstrating loss of flow voids, and $\mathbf{b}$ subject $B$ demonstrating maintained flow voids, at the skull base (red circles). Subject A demonstrated lower white matter (white circle) b50 ADC values than the white matter (white circle) in subject B ( 0.13 vs $\left.0.92 \times 10^{-3} \mathrm{~mm}^{2} / \mathrm{s}\right)$. The gray matter (green circles) was markedly low in both subjects, although lower in the subject without flow voids ( 0.12 vs. $\left.0.47 \times 10^{-3} \mathrm{~mm}^{2} / \mathrm{s}\right)$. Both subjects had clinical confirmation of BD per AAN criteria, suggesting that gray matter perfusion values were more predictive of $\mathrm{BD}$ than the absence of flow voids

Low b-value DWI is promising for differentiation of BD and HIE with maintained perfusion. This sequence can potentially provide important prognostic information to clinicians and families and aid in critical care decisions. The utility and low-maintenance ability of b50 DWI to demonstrate perfusion abnormalities demonstrate its promise for use in the setting of cardiorespiratory arrest.

\section{Conclusion}

Low b-value DWI is promising for the diagnosis of brain death and HIE after cardiorespiratory arrest. This method demonstrated perfect prediction of brain death using a cutoff ADC value of $<1.085 \times 10^{-3} \mathrm{~mm}^{2} / \mathrm{s}$ in the gray matter. Low b50 ADC values in the gray matter were more predictive of brain death than in the white matter.

\section{Abbreviations}

ADC: Apparent diffusion coefficient; ASL: Arterial spin labeling; BD: Brain death; CNS: Central nervous system; CPC: Cerebral Performance Category; CSF: Cerebrospinal fluid; DWI: Diffusion weighted imaging; HIE: Hypoxic ischemic encephalopathy; IVIM: Intravoxel incoherent motion; MPRAGE: Magnetization prepared rapid acquisition gradient echo; MRI: Magnetic resonance imaging; ROSC: Return of spontaneous circulation SPECT: Single photon emission computed tomography

\section{Availability of data and materials}

The datasets used and/or analyzed during the current study are available from the corresponding author on reasonable request.

\section{Authors' contributions}

MEP: conception, study design, data analysis, methodology, data collection, authoring, editing. JSA: study design, data analysis, methodology, authoring, editing. UAR: data analysis, methodology, editing. LMS: data analysis, methodology, editing. PJH: data analysis, editing. AdH: methodology, data analysis, editing. SEK: methodology, editing. JSM: conception, study design data analysis, methodology, data collection, authoring, editing. All authors read and approved the final manuscript.

\section{Ethics approval and consent to participate}

This study was approved by our Institutional Review Board. Due to the retrospective nature of this study, informed consent was waived.

\section{Consent for publication}

Images included are entirely unidentifiable and there are no details on individuals reported within the manuscript.

\section{Competing interests}

The authors declare that they have no competing interests.

\section{Publisher's Note}

Springer Nature remains neutral with regard to jurisdictional claims in published maps and institutional affiliations.

\section{Author details}

'Department of Radiology and Imaging Sciences, University of Utah, Salt Lake City, UT, USA. ${ }^{2}$ Department of Neurology, University of Utah, Salt Lake City, UT, USA. ${ }^{3}$ Utah Center for Advanced Imaging Research, Department of Radiology, University of Utah, Salt Lake City, UT, USA. ${ }^{4}$ Department of Radiology and Imaging Sciences, University of Utah Health Sciences Center, 30 North, 1900 East \#1A071, Salt Lake City, UT 84132-2140, USA.

Received: 23 March 2018 Accepted: 30 May 2018

Published online: 20 June 2018

\section{References}

1. Garrett MP, Williamson RW, Bohl MA, Bird CR, Theodore N. Computed tomography angiography as a confirmatory test for the diagnosis of brain death. J Neurosurg. 2018;128(2):639-44. 
2. Lopez-Navidad A, Caballero F, Domingo P, Marruecos L, Estorch M, Kulisevsky J, Mora J. Early diagnosis of brain death in patients treated with central nervous system depressant drugs. Transplantation. 2000;70:131-5.

3. Ramjug S, Hussain N, Yonan N. Prolonged time between donor brain death and organ retrieval results in an increased risk of mortality in cardiac transplant recipients. Interact Cardiovasc Thorac Surg. 2011;12:938-42.

4. Taccone F, Cronberg T, Friberg H, Greer D, Horn J, Oddo M, Scolletta S, Vincent $\mathrm{JL}$. How to assess prognosis after cardiac arrest and therapeutic hypothermia. Crit Care. 2014;18:202.

5. Perman SM, Kirkpatrick JN, Reitsma AM, Gaieski DF, Lau B, Smith TM, Leary M, Fuchs BD, Levine JM, Abella BS, et al. Timing of neuroprognostication in postcardiac arrest therapeutic hypothermia. Crit Care Med. 2012;40:719-24

6. Bernat JL. On irreversibility as a prerequisite for brain death determination. Adv Exp Med Biol. 2004;550:161-7.

7. Sohn CH, Lee HP, Park JB, Chang HW, Kim E, Kim E, Park UJ, Kim HT, Ku J. Imaging findings of brain death on 3-tesla MRI. Korean J Radiol. 2012;13:541-9.

8. Young GB, Shemie SD, Doig CJ, Teitelbaum J. Brief review: the role of ancillary tests in the neurological determination of death. Can J Anaesth. 2006:53:620-7.

9. Heran MK, Heran NS, Shemie SD. A review of ancillary tests in evaluating brain death. Can J Neurol Sci. 2008;35:409-19.

10. Wieler H, Marohl K, Kaiser KP, Klawki P, Frossler H. Tc-99m HMPAO cerebral scintigraphy. A reliable, noninvasive method for determination of brain death. Clin Nucl Med. 1993;18:104-9.

11. Orrison WW Jr, Champlin AM, Kesterson OL, Hartshorne MF, King JN. MR 'hot nose sign' and 'intravascular enhancement sign' in brain death. AJNR Am J Neuroradiol. 1994;15:913-6.

12. Sinha P, Conrad GR. Scintigraphic confirmation of brain death. Semin Nucl Med. 2012;42:27-32

13. Ziegler D, Cravens G, Poche G, Gandhi R, Tellez M. Use of transcranial Doppler in patients with severe traumatic brain injuries. J Neurotrauma. 2017;34:121-7.

14. Chang JJ, Tsivgoulis G, Katsanos AH, Malkoff MD, Alexandrov AV. Diagnostic accuracy of transcranial Doppler for brain death confirmation: systematic review and meta-analysis. AJNR Am J Neuroradiol. 2016:37:408-14.

15. Tong KA, Ashwal S, Obenaus A, Nickerson JP, Kido D, Haacke EM. Susceptibility-weighted MR imaging: a review of clinical applications in children. AJNR Am J Neuroradiol. 2008;29:9-17.

16. Kang KM, Yun TJ, Yoon BW, Jeon BS, Choi SH, Kim JH, Kim JE, Sohn CH, Han $\mathrm{MH}$. Clinical utility of arterial spin-labeling as a confirmatory test for suspected brain death. AJNR Am J Neuroradiol. 2015:36:909-14.

17. Haller S, Badoud S, Nguyen D, Barnaure I, Montandon ML, Lovblad KO, Burkhard PR. Differentiation between Parkinson disease and other forms of parkinsonism using support vector machine analysis of susceptibilityweighted imaging (SWI): initial results. Eur Radiol. 2013;23:12-9.

18. Heursen EM, Zuazo Ojeda A, Benavente Fernandez I, Jimenez Gomez G, Campuzano Fernandez-Colima R, Paz-Exposito J, Lubian Lopez SP. Prognostic value of the apparent diffusion coefficient in newborns with hypoxic-ischaemic encephalopathy treated with therapeutic hypothermia. Neonatology. 2017;112:67-72.

19. Wijman CA, Mlynash M, Caulfield AF, Hsia AW, Eyngorn I, Bammer R, Fischbein N, Albers GW, Moseley M. Prognostic value of brain diffusionweighted imaging after cardiac arrest. Ann Neurol. 2009;65:394-402.

20. Lovblad KO, Bassetti C. Diffusion-weighted magnetic resonance imaging in brain death. Stroke. 2000;31:539-42.

21. Federau C, Maeder P, O'Brien K, Browaeys P, Meuli R, Hagmann P. Quantitative measurement of brain perfusion with intravoxel incoherent motion MR imaging. Radiology. 2012;265:874-81.

22. lima M, Le Bihan D. Clinical intravoxel incoherent motion and diffusion MR imaging: past, present, and future. Radiology. 2016;278:13-32.

23. Le Bihan D, Breton E, Lallemand D, Aubin ML, Vignaud J, Laval-Jeantet M. Separation of diffusion and perfusion in intravoxel incoherent motion MR imaging. Radiology. 1988;168:497-505.

24. Federau C, O'Brien K, Meuli R, Hagmann P, Maeder P. Measuring brain perfusion with intravoxel incoherent motion (IVIM): initial clinical experience. J Magn Reson Imaging. 2014;39:624-32.

25. Lemke A, Stieltjes B, Schad LR, Laun FB. Toward an optimal distribution of b values for intravoxel incoherent motion imaging. Magn Reson Imaging. 2011:29:766-76.

26. Federau C, Sumer S, Becce F, Maeder P, O'Brien K, Meuli R, Wintermark M. Intravoxel incoherent motion perfusion imaging in acute stroke: initial clinical experience. Neuroradiology. 2014;56:629-35.
27. Federau C, Cerny M, Roux M, Mosimann PJ, Maeder P, Meuli R, Wintermark M. IVIM perfusion fraction is prognostic for survival in brain glioma. Clin Neuroradiol. 2017;27(4):485-92.

28. Marzi S, Piludu F, Forina C, Sanguineti G, Covello R, Spriano G, Vidiri A Correlation study between intravoxel incoherent motion MRI and dynamic contrast-enhanced MRI in head and neck squamous cell carcinoma: evaluation in primary tumors and metastatic nodes. Magn Reson Imaging. 2017;37:1-8.

29. Gao QQ, Lu SS, Xu XQ, Wu CJ, Liu XL, Liu S, Shi HB. Quantitative assessment of hyperacute cerebral infarction with intravoxel incoherent motion MR imaging: initial experience in a canine stroke model. J Magn Reson Imaging. 2017:46(2):550-6

30. Suo S, Cao M, Zhu W, Li L, Li J, Shen F, Zu J, Zhou Z, Zhuang Z, Qu J, et al. Stroke assessment with intravoxel incoherent motion diffusion-weighted MRI. NMR Biomed. 2016;29:320-8.

31. Zhang CE, Wong SM, Uiterwijk R, Staals J, Backes WH, Hoff El, Schreuder T, Jeukens CR, Jansen JF, van Oostenbrugge RJ. Intravoxel incoherent motion imaging in small vessel disease: microstructural integrity and microvascular perfusion related to cognition. Stroke. 2017;48:658-63.

32. Hu LB, Hong N, Zhu WZ. Quantitative measurement of cerebral perfusion with Intravoxel incoherent motion in acute ischemia stroke: initial clinical experience. Chin Med J. 2015;128:2565-9.

33. Conklin J, Heyn C, Roux M, Cerny M, Wintermark M, Federau C. A simplified model for intravoxel incoherent motion perfusion imaging of the brain. AJNR Am J Neuroradiol. 2016;37:2251-7.

34. Kim HS, Suh CH, Kim N, Choi CG, Kim SJ. Histogram analysis of intravoxel incoherent motion for differentiating recurrent tumor from treatment effect in patients with glioblastoma: initial clinical experience. AJNR Am J Neuroradiol. 2014;35:490-7.

35. Wijdicks EF, Varelas PN, Gronseth GS, Greer DM. Evidence-based guideline update: determining brain death in adults: report of the quality standards Subcommittee of the American Academy of neurology. Neurol. 2010;74:1911-8.

36. Lee JH, Cheong H, Lee SS, Lee CK, Sung YS, Huh JW, Song JA, Choe H. Perfusion assessment using intravoxel incoherent motion-based analysis of diffusion-weighted magnetic resonance imaging: validation through phantom experiments. Investig Radiol. 2016:51:520-8.

37. Alsop DC, Detre JA. Reduced transit-time sensitivity in noninvasive magnetic resonance imaging of human cerebral blood flow. J Cereb Blood Flow Metab. 1996;16:1236-49.

38. Shaikh H, Lechpammer M, Jensen FE, Warfield SK, Hansen AH, Kosaras B, Shevell M, Wintermark $P$. Increased brain perfusion persists over the first month of life in term asphyxiated newborns treated with hypothermia: does it reflect activated angiogenesis? Transl Stroke Res. 2015;6:224-33.

39. Wintermark P, Hansen A, Gregas MC, Soul J, Labrecque M, Robertson RL, Warfield SK. Brain perfusion in asphyxiated newborns treated with therapeutic hypothermia. AJNR Am J Neuroradiol. 2011;32:2023-9.

40. Wintermark P, Moessinger AC, Gudinchet F, Meuli R. Perfusion-weighted magnetic resonance imaging patterns of hypoxic-ischemic encephalopathy in term neonates. J Magn Reson Imaging. 2008;28:1019-25.

41. Wintermark P, Moessinger AC, Gudinchet F, Meuli R. Temporal evolution of MR perfusion in neonatal hypoxic-ischemic encephalopathy. J Magn Reson Imaging. 2008;27:1229-34

42. De Vis JB, Hendrikse J, Petersen ET, de Vries LS, van Bel F, Alderliesten T, Negro S, Groenendaal F, Benders MJ. Arterial spin-labelling perfusion MRI and outcome in neonates with hypoxic-ischemic encephalopathy. Eur Radiol. 2015:25:113-21.

43. de Havenon A, Sultan-Qurraie A, Tirschwell D, Cohen W, Majersik J, Andre JB. Medial occipital lobe hyperperfusion identified by arterial spin-labeling: a poor prognostic sign in patients with hypoxic-ischemic encephalopathy. AJNR Am J Neuroradiol. 2015;36:2292-5. 